\title{
Acetylpuerarin increases cell viability and reduces apoptosis in rat hippocampal neurons following oxygen-glucose deprivation/reperfusion
}

\author{
DONG-MEI LIU ${ }^{1}$, ZHEN-HUI WANG ${ }^{1}$, LI LIU $^{1}$, XIU-MEI ZHANG ${ }^{2}$ and FENG-LAN LOU ${ }^{1}$ \\ ${ }^{1}$ School of Nursing; ${ }^{2}$ Department of Pharmacology, School of Medicine, \\ Shandong University, Jinan, Shandong 250012, P.R. China
}

Received May 27, 2013; Accepted August 30, 2013

DOI: $10.3892 / \mathrm{mmr} .2013 .1671$

\begin{abstract}
The effects of acetylpuerarin treatment following oxygen-glucose deprivation/reperfusion (OGD/R) were examined in rat hippocampal neurons in vitro and compared with the effects of acetylpuerarin in normoxic cells to confirm acetylpuerarin's potential neuroprotective effects, including apoptosis inhibition. Wistar rat embryo hippocampal cells (day 18, E18) cultured for 8 days were subjected to 3 h OGD treatment, followed by reperfusion for 12,24 or $36 \mathrm{~h}$. For each time interval, a group of cells was left untreated (OGD/R-only groups) and treated with $0.1,0.4$ and $1.6 \mu \mathrm{M}$ acetylpuerarin (OGD/R+acetylpuerarin). Neuron viability, apoptosis and caspase- 8 and -3 activities were assessed by the reduction of 3-(4,5-dimethylthiazol-2-yl)-2,5-diphenyltetrazolium bromide (MTT), 4',6-diamidino-2-phenylindole (DAPI) and terminal deoxynucleotidyl-transferase-mediated dUTP nick end labeling (TUNEL) and spectrophotometric assays, respectively. Fas-ligand (Fas-L), Fas-associated death domain (FADD) and tumor necrosis factor- $\alpha$ (TNF- $\alpha$ ) were determined by western blot analysis. Compared with control cells, OCD/R+acetylpuerarin cells treated with $0.1,0.4$ and $1.6 \mu \mathrm{M}$ doses showed a concentration-dependent increase in hippocampal cell survival and viability by $69.93 \pm 2.28 \%$, $81.49 \pm 2.13 \%$ and $85.28 \pm 2.38 \%$ at $12 \mathrm{~h}, 68.59 \pm 3.02 \%$, $77.85 \pm 2.84 \%$ and $85.64 \pm 4.39 \%$ at $24 \mathrm{~h}$ and $69.70 \pm 1.70 \%$, $77.21 \pm 3.21 \%$ and $83.90 \pm 2.12 \%$ at $36 \mathrm{~h}(\mathrm{P}<0.05)$. Furthermore, OCD/R+acetylpuerarin cells exhibited a dose-dependent decrease in caspase- 8 and -3 activation, TUNEL and DAPI-positive neurons and Fas-L, FADD and TNF- $\alpha$ expression. In conclusion, acetylpuerarin protects against OGD/R-induced neuronal apoptosis predominantly in the first
\end{abstract}

Correspondence to: Professor Feng-Lan Lou, School of Nursing, Shandong University, 44 Wenhua Xi Road, Jinan, Shandong 250012, P.R. China

E-mail: to506@163.com

Key words: oxygen-glucose deprivation/reperfusion, acetylpuerarin, apoptosis
$24 \mathrm{~h}$ following ischemia, which may be useful in mediating neuronal apoptosis in ischemic stroke patients.

\section{Introduction}

Cerebrovascular disease-related stroke affects $\leq 300$ of every 100,000 members of the Chinese population (1) and the most common form of stroke, ischemic stroke, remains a leading cause of mortality and long-term disability in aging patients (2). Over the course of as little as $30 \mathrm{~min}$, ischemia begins to produce oxygen-glucose deprivation (OGD)-induced neuronal apoptosis that may progress rapidly with increasing ischemia duration (3). Thus, the primary goal of the majority of contemporary medicines for stoke is the mediation of OGD-induced neuronal apoptosis during cerebral ischemia/reperfusion (4). Acetylpuerarin is a novel compound that has been reported to effectively attenuate the morphological changes leading to apoptosis in hippocampal cells following cerebral ischemia/reperfusion, with potential benefits even when applied a number of hours following the initial ischemic event (5). Unlike the extremely small treatment window $(\sim 3 \mathrm{~h})$ of current United States Food and Drug Administration-approved therapies for stroke, including tissue plasminogen activator (6-8), acetylpuerarin may provide an alternative for stroke treatment at advanced stages (9). Prior to clinical implementation, a greater understanding of the dose-effects and mechanistic action of acetylpuerarin on hippocampal cell apoptosis during ischemia/reperfusion is required.

Acetylpuerarin is a modern synthetic derivative of puerarin (daidzein-8-C-glucoside) with higher lipid solubility than the naturally occurring compound, allowing it to better penetrate the blood-brain barrier (Fig. 1). Puerarin is an isoflavonoid compound used for treating cardiovascular disorders derived from the Chinese medical herb Radix puerariae, known as ' $\mathrm{Ge}$ Gen', from the root of the kudzu plant. Treatment of puerarin has been reported to effectively reduce symptoms following acute ischemic stroke with few side effects in a number of preliminary in vivo and in vitro studies (10-12). A number of studies have reported that acetylpuerarin exerts protective effects against ischemia-reperfusion injury in the hippocampus by mediating the cascade of events leading to OGD-induced damage $(5,13,14)$. Thus, acetylpuerarin may be capable of mediating the degree 
of irreversible injury caused by neuronal cell death following OGD/reperfusion (OGD/R), a complex, synergistic process in which proliferation of reactive oxygen species and free radicals, induction of tumor necrosis factor- $\alpha$ (TNF- $\alpha$ ) and other inflammatory factors, release of cytokines, excitotoxicity, unbalanced activites of caspase- 8 and -3 , and abnormalities in calcium levels contribute to apoptosis (9). The neuroprotective effects of puerarin and acetylpuerarin on cerebral ischemic damage are associated with the inhibition of inflammatory factor TNF- $\alpha$ and apoptosis (active caspase-3), free radical scavenging or antioxidative activity (15). However, the full mechanism of acetylpuerarin action by dosage has not been characterized.

The current study examines the detailed mechanisms underlying the neuroprotective effects of acetylpuerarin in hippocampal neurons by dose, including the effects on caspase- 8 and -3 activities associated with apoptosis, Fas-ligand (Fas-L), Fas-associated death domain (FADD) and TNF- $\alpha$ inflammatory factors and neuronal cell viability in OGD/R-induced hippocampal neurons. The observations of the resultant injury and neuroprotective mechanisms lead to an improved mechanistic understanding of acetylpuerarin that provides an essential basis for potential clinical applications of this drug in ischemic stroke patients.

\section{Materials and methods}

Animal subjects and study design. Hippocampal cells were isolated from Wistar rat embryos (day 18, E18) purchased from the Laboratory Animal Center of the Shandong University (Shandong, China; Grade II, Certificate No. 20021015) and cultured for 8 days.

Cells were subjected to $3 \mathrm{~h}$ OGD treatment followed by reperfusion for 12, 24 or $36 \mathrm{~h}$. For each time interval, a group of cells was left untreated (OGD/R groups) and treated with $0.1,0.4$ and $1.6 \mu \mathrm{M}$ acetylpuerarin (OGD/R+acetylpuerarin) [13 groups: control (I); OGD/R 12, 24 or 36 h -only (II, VI, X) and OGD/R 12, 24 or $36 \mathrm{~h}+0.1,0.4$ and $1.6 \mu \mathrm{M}$ acetylpuerarin (III-V, VII-IX and XI-XIII), respectively]. The effects and mechanisms of acetylpuerarin on hippocampal neuron apoptosis in OGD/R and normoxic cells were observed. The study was approved by Shandong University Ethical Committee and animals were used in accordance with all relevant guidelines for animal use and care.

Primary embryonic hippocampal neuron cultures. Hippocampi were dissected from E18 rat brains and sampled primary embryonic hippocampal neuron cells were seeded onto 96-well tissue culture plates coated with poly-D-Lysine (Sigma-Aldrich, St. Louis, MO, USA) at $5 \times 10^{5}$ cells $/ \mathrm{ml}$ or on 6 -well tissue culture plates at $1 \times 10^{6}$ cells $/ \mathrm{ml}$, as previously described (16). Neurons were cultured in a medium containing neurobasal medium (Gibco-BRL, Carlsbad, CA, USA), 2\% B27 (Gibco-BRL), 2 mM glutamine (Sigma -Aldrich), $50 \mathrm{U} / \mathrm{ml}$ penicillin $\mathrm{G}$ and $50 \mu \mathrm{g} / \mathrm{ml}$ streptomycin (Gibco-BRL) in a humidified incubator with $5 \% \mathrm{CO}_{2}$ at $37^{\circ} \mathrm{C}$. Half of the cultured medium was changed at 3 day intervals and cells were sampled for use in the following experiments at 8 days.

OGD/R treatment. Cells in the OGD/R-only and OGD/R+acetylpuerarin groups underwent OGD, as previously described (17). Briefly, experimental cell culture medium was replaced with glucose-free Hanks' balanced salt solution (Invitrogen Life Technologies, Carlsbad, CA, USA) and cells were placed in an anaerobic chamber $\left(95 \% \mathrm{~N} 2 / 5 \% \mathrm{CO}_{2}\right)$ for $180 \mathrm{~min}$, following which OGD was terminated by removing the cultures from the anaerobic chamber, replacing deoxygenated and glucose-free Hanks' BSS with pre-OGD culture medium without B27 supplement and returning cells to normoxic conditions. Reperfusion was conducted for 12, 24 or $36 \mathrm{~h}$. Control group cells were similarly incubated in Hanks' BSS with $10 \mathrm{mM}$ glucose in a normoxic incubator.

Acetylpuerarin administration. Acetylpuerarin was dissolved in DMSO $\leq 0.1 \%$ and administered at the onset of the ischemic period in concentrations of $0.1,0.4$ and $1.6 \mu \mathrm{M}$ in the OGD/R+acetylpuerarin 9 groups III-V, VII-IX and XI-XIII, respectively, according to the dosage concentrations suggested by Liu et al (5). Each experiment was performed in cultures from three E18 rats and was repeated in triplicate.

\section{3-(4,5-dimethylthiazol-2-yl)-2,5-diphenyltetrazolium}

bromide (MTT) assay. Upon completion of reperfusion, $20 \mu \mathrm{l} /$ well of MTT was added at a final concentration of $0.5 \mathrm{mg} / \mathrm{ml}$. Cultures were allowed to incubate at $37^{\circ} \mathrm{C}$ and $5 \% \mathrm{CO}_{2}$ for $4 \mathrm{~h}$. The culture medium was aspirated and replaced with $150 \mu \mathrm{l} /$ well of DMSO. The optical density (OD) was measured at a test wavelength of $492 \mathrm{~nm}$ in a microplate reader (Thermo MK3; Thermo Fisher Scientific, Shanghai, China). The following formula (18) was used to calculate cell viability as percentage: (absorbance of treated cells / absorbance of normal cells) x $100 \%$.

4',6-diamidino-2-phenylindole (DAPI) staining. Upon completion of reperfusion, cultured neurons were incubated with $5 \mu \mathrm{g} / \mathrm{ml}$ fluorescent DNA-binding dye DAPI (Sigma-Aldrich) at $37^{\circ} \mathrm{C}$ for $1 \mathrm{~min}$. Staining solution was removed and apoptotic cells were visualized by fluorescence microscopy (Olympus DP-72; Olympus Corporation, Tokyo, Japan). Apoptotic cells were morphologically identified by cytoplasmic or nuclear shrinkage, chromatin condensation and DNA fragmentation. The percentage of apoptotic cells was determined in 5 randomly selected fields of $\sim 500$ neurons per field (magnification, x100). Experimental data was pooled from three coverslips and each experiment was conducted three times.

Terminal deoxynucleotidyl-transferase-mediated dUTP nick end labeling (TUNEL) staining. Neuronal apoptosis was detected using a TUNEL assay $24 \mathrm{~h}$ following OGD initiation. TUNEL-positive (apoptotic) cells appeared dark brown. These cells were counted in 5 randomly selected microscopic fields (Olympus DP-71; Olympus Corporation) (magnification, $\mathrm{x} 100$ ). Apoptotic index (AI) was calculated as: (apoptotic neurons / total neurons) x $100 \%$.

Western blot analysis for Fas-L, FADD and TNF- $\alpha$ expression. Total protein $(50 \mu \mathrm{g})$ was extracted from samples from each group, separated by $12 \%$ sodium dodecyl sulfate polyacrylamide gel electrophoresis and blotted onto polyvinylidene fluoride membranes (Beijing Solarbio Science and 
Technology Co. Ltd., Beijing, China). Membranes were probed with primary antibodies against Fas-L (1:400), FADD (1:500) and TNF- $\alpha$ (1:300; Santa Cruz Biotechnology, Inc., Santa Cruz, CA, USA) and peroxidase-conjugated secondary antibody (1:5,000; ZSGB-Bio, Beijing, China). $\beta$-actin (1:2,000; ZSGB-Bio) was used as a control. Band intensities were quantitated using AlphaEaseFC software (Genetic Technologies, Miami, FL, USA).

Caspase- 8 and -3 activity assay. Caspase- 8 and -3 activities were assessed using FLICE/caspase-8 and caspase-3/CPP32 colorimetric assay kits, respectively, (BioVision, Inc., Milpitas, CA, USA), according to the instructions suggested by the manufacturer. Briefly, 100 or $50 \mu \mathrm{g}$ of total protein were incubated with $10 \mathrm{mM}$ dithiothreitol and IETD-pNA for caspase- 8 and -3 activity quantification (caspase- 8 substrate; $200 \mu \mathrm{M}$ final concentration) or MDEVD-pNA (caspase-3 substrate; $200 \mu \mathrm{M}$ final concentration) at $37^{\circ} \mathrm{C}$ for $2 \mathrm{~h}$. Samples were determined at $405 \mathrm{~nm}$ in a microplate reader (Therm MK3; Finland). The final reading did not include the background reading of cell lysates and buffers. Enzymatic activity was expressed in arbitrary units of OD per mg protein.

Statistical analysis. All data were analyzed using SPSS version 16 (SPSS, Inc., Chicago, IL, USA) and expressed as means \pm SEM. Between group and multiple group differences were analyzed using Student's t-tests. $\mathrm{P}<0.05$ was considered to indicate a statistically significant difference.

\section{Results}

Acetylpuerarin increases neuron viability following $O G D / R$. The MTT assay revealed no significant changes in viability in the control and normoxic control groups treated with acetylpuerarin treatment at all times $(\mathrm{P}>0.05$, data not shown). Compared with the OGD/R-only group, cell survival was $61.94 \pm 2.73 \%(\mathrm{OGD} / \mathrm{R}, 12 \mathrm{~h}), 60.61 \pm 3.29 \%(\mathrm{OGD} / \mathrm{R}$, $24 \mathrm{~h})$ and $57.77 \pm 0.66 \%(\mathrm{OGD} / \mathrm{R}, 36 \mathrm{~h})$. Compared with the control group, OGD/R+acetylpuerarin groups treated with acetylpuerarin doses of $0.1,0.4$ and $1.6 \mu \mathrm{M}$ increased cell survival and viability by $69.93 \pm 2.28 \%, 81.49 \pm 2.13 \%$ and $85.28 \pm 2.38 \%$ at $12 \mathrm{~h}$, respectively; $68.59 \pm 3.02 \%, 77.85 \pm 2.84 \%$ and $85.64 \pm 4.39 \%$ at $24 \mathrm{~h}$, respectively and $69.70 \pm 1.70 \%$, $77.21 \pm 3.21 \%$ and $83.90 \pm 2.12 \%$ at $36 \mathrm{~h}$, respectively $(\mathrm{P}<0.05$; Fig. 2). Higher acetylpuerarin concentrations enhanced neuron survival more efficiently in a concentration-dependent manner. Similar results were obtained by morphological analysis. The control group exhibited round cell bodies with clear edges and fine dendritic network. OGD/R groups exhibited a decreasing number of neurons, shrinkage of cell bodies and disruption of dendritic networks. Acetylpuerarin mitigated the morphological manifestations of cell damage (data not shown).

Acetylpuerarin reduces apoptosis following $O D G / R$. DAPI staining revealed that $<8 \%$ of control-only cells showed signs of apoptosis at any time point; however, OGD/R treatment resulted in significant time-dependent increases in apoptotic cell numbers, peaking at $24 \mathrm{~h}(\mathrm{P}<0.01$; Fig. 3). In the OGD/R-only group at $24 \mathrm{~h}, 75.85 \pm 7.59 \%$ of
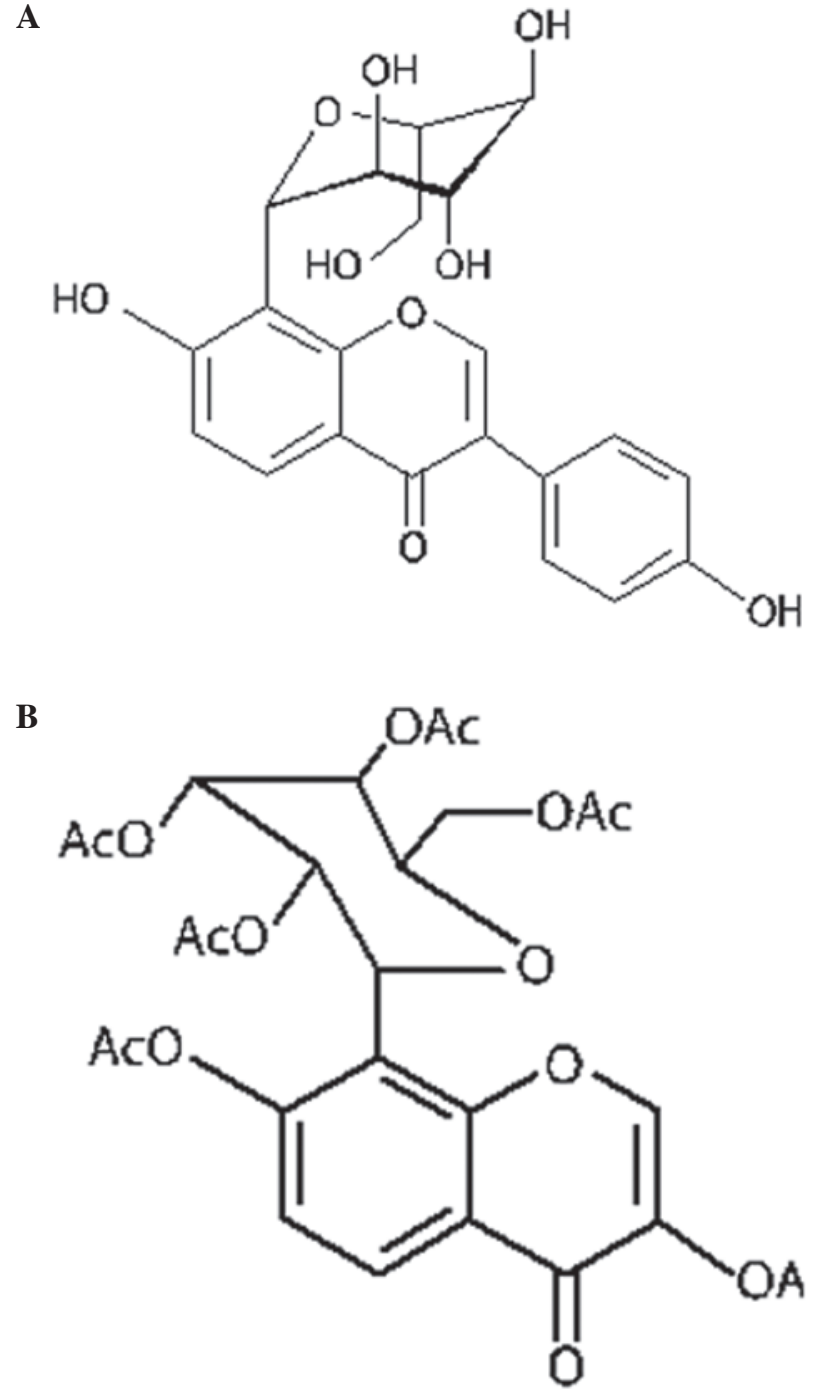

Figure 1. Structure of (A) puerarin and (B) acetylpuerarin. Puerarin is found in traditional Chinese medicine and acetylpuerarin is a synthetic derivative with enhanced lipid solubility.

remaining cells showed signs of apoptosis. Comparatively, OGD/R+acetylpuerarin groups treated with $0.4 \mu \mathrm{M}$ acetylpuerarin doses exhibited reductions in DAPI positive neurons to $63.01 \pm 7.35 \%, 59.06 \pm 5.98 \%$ and $51.05 \pm 5.98 \%$, respectively (all $\mathrm{P}<0.05$ ). Thus, increasing the concentration of acetylpuerarin exhibited no significant effects on apoptosis rates in cells treated with OGD/R following $24 \mathrm{~h}$ reperfusion (all $\mathrm{P}>0.05$ ).

These results were confirmed with TUNEL staining, indicating that $5 \%$ of the control-only group cells exhibited apoptosis under basal conditions. TUNEL-positive cells were markedly increased in the OGD/R-only group at $24 \mathrm{~h}$ and the AI was $68.79 \pm 10.01 \%$. Acetylpuerarin treatment decreased the number of TUNEL-positive cells to $52.30 \pm 9.73 \%$, $46.08 \pm 13.42 \%$ and $39.04 \pm 7.29 \%$ in all OGD/R+acetylpuerarin groups treated with $24 \mathrm{~h}$ reperfusion (all $\mathrm{P}<0.05$; Table I).

Acetylpuerarin inhibited OGD/R-induced caspase-8 and -3 activation. Caspase- 8 was significantly increased in hippocampal neurons following OGD/R treatments at $24 \mathrm{~h}$ and acetylpuerarin treatment led to a concentration-dependent decrease in the expression of enzymatic activity of caspase- 8 
Table I. Effects of acetylpuerarin on AI (24-h reperfusion).

\begin{tabular}{lcc}
\hline Group & $\begin{array}{c}\text { Acetylpuerarin } \\
\text { concentration, } \mu \mathrm{M}\end{array}$ & AI, \% \\
\hline Control & 0 & $4.57 \pm 2.48$ \\
OGD/R & 0 & $68.79 \pm 10.06^{\mathrm{a}}$ \\
OGD/R+acetylpuerarin ${ }^{\mathrm{b}}$ & & \\
VII & 0.1 & $52.30 \pm 9.73^{\mathrm{c}}$ \\
VIII & 0.4 & $46.08 \pm 13.42^{\mathrm{c}}$ \\
IX & 1.6 & $39.04 \pm 7.29^{\mathrm{c}}$ \\
\hline
\end{tabular}

${ }^{\mathrm{a}} \mathrm{P}<0.05$ vs. control-only group. ${ }^{\mathrm{b}}$ Treated with $3 \mathrm{~h}$ oxygen-glucose deprivation and $24 \mathrm{~h}$ reperfusion. ${ }^{\mathrm{c}} \mathrm{P}<0.01$, vs. control-only group. Values are expressed as the mean $\pm \mathrm{SD}$ of three independent experiments. AI, apoptotic index; OGD/R, oxygen-glucose deprivation/reperfusion.

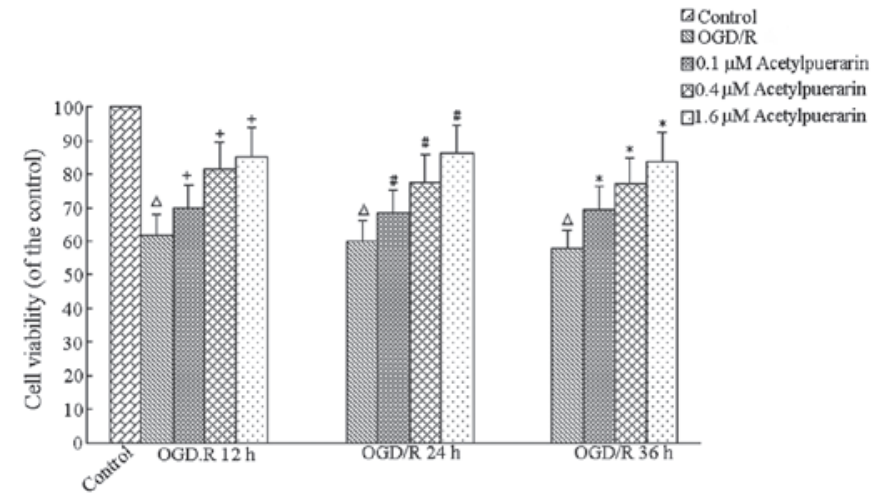

Figure 2. Effects of acetylpuerarin on the viability of OGD/R injured hippocampal neurons. Cell viability was assessed by MTT. Data are expressed as the mean $\pm \mathrm{SD}$ of three replicates. ${ }^{\Delta} \mathrm{P}<0.01$, vs. control-only; ${ }^{+} \mathrm{P}<0.05$, vs. OGD/R $12 \mathrm{~h} ;{ }^{*} \mathrm{P}<0.05$, vs. OGD/R $24 \mathrm{~h}$; and "P<0.01, vs. OGD/R $36 \mathrm{~h}$. OGD/R, oxygen-glucose deprivation/reperfusion; MTT, 3-(4,5-dimethylthiazol-2-yl)-2,5-diphenyltetrazolium bromide.

(Table II). As caspase-3 is considered to be a prototypical caspase and a key effector of programmed cell death (19), enzymatic activity of caspase-3 was also determined. As shown in Table II, exposure to OGD significantly enhanced caspase-3 proteolytic activity by $24 \mathrm{~h}$, while acetylpuerarin significantly diminished the activation of caspase- 3 activity in a concentration-dependent manner.

Acetylpuerarin inhibited OGD/R-induced Fas-L, FADD and $T N F-\alpha$ protein expression. Western blot analysis indicated that Fas-L levels were induced by OGD treatment in hippocampal neurons by $24 \mathrm{~h}$ post-reperfusion (Fig. 4A). This Fas-L induction was significantly attenuated by acetylpuerarin treatment in a concentration-dependent manner. High levels of FADD protein were detected in neurons following OGD/R $24 \mathrm{~h}$. However, FADD expression was significantly downregulated by acetylpuerarin (Fig. 4B). OGD/R-induced apoptosis was accompanied by a significant increase in TNF- $\alpha$ (Fig. 4C). Western blot analysis also revealed that acetylpuerarin inhibited TNF- $\alpha$ expression induced by OGD/R $24 \mathrm{~h}$ in a dose-dependent manner.
A

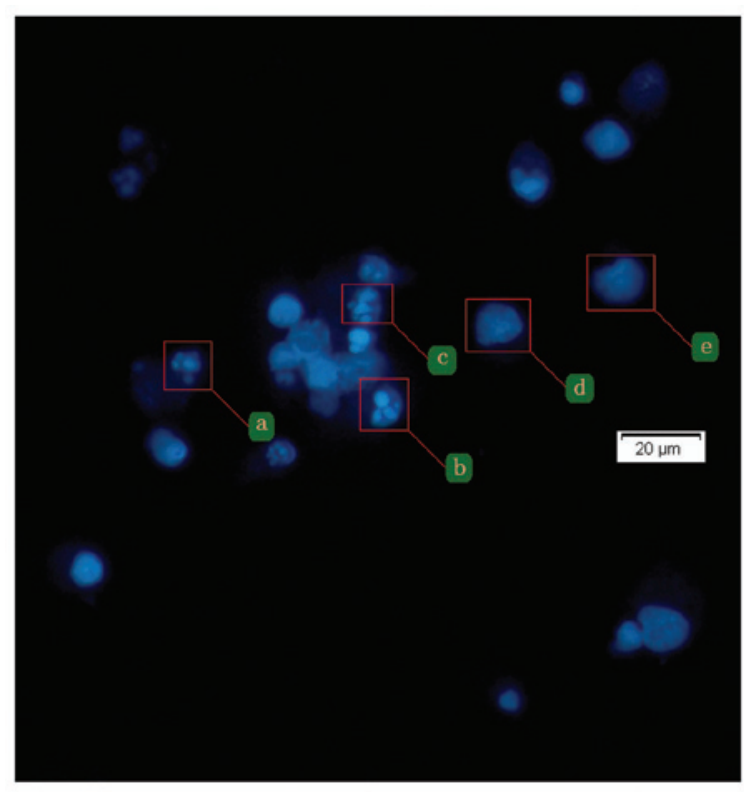

B

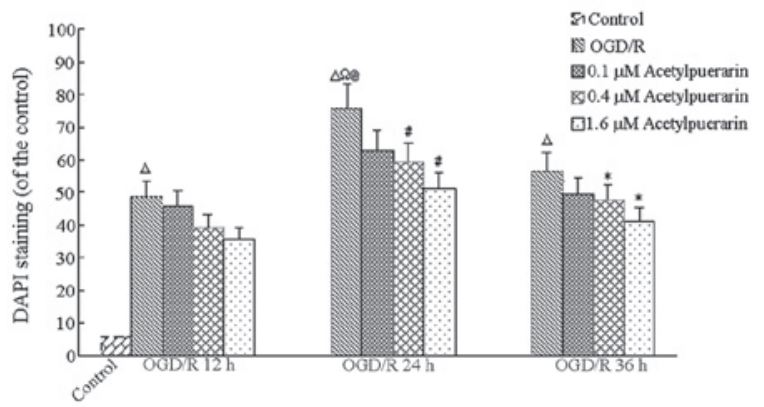

Figure 3. Effect of acetylpuerarin on DAPI staining of hippocampal cultures following OGD/R treatment. (A) Nuclear morphological changes in neurons under fluorescence microscopy (blue indicates nuclear stained by DAPI). (a-c) Apoptotic cells and (d-e) normal cells are indicated (magnification, $\mathrm{x} 400$ ). (B) DAPI staining of hippocampal cultures. Values are expressed as the means $\pm \mathrm{SD}$ of three independent experiments. ${ }^{\Delta} \mathrm{P}<0.01$, vs control-only; ${ }^{\Omega} \mathrm{P}<0.05$, vs. OGD/R $12 \mathrm{~h} ;{ }^{\circledR} \mathrm{P}<0.05$, vs. OGD/R $36 \mathrm{~h} ;{ }^{\prime \prime} \mathrm{P}<0.01$, vs. OGD/R 24 h; ${ }^{*} \mathrm{P}<0.05$, vs. OGD/R 36 h. OGD/R, oxygen-glucose deprivation/reperfusion.

\section{Discussion}

A model of ischemic OGD/R in hippocampal neurons was used to assess the potential protective role of acetylpuerarin in ischemic injury. Acetylpuerarin not only increased neuron viability, but also decreased DAPI and TUNEL-positive cells, caspase- 8 and -3 activity and Fas-L protein expression, FADD and TNF- $\alpha$ induced by OGD/R. Thus, acetylpuerarin may be capable of mediating the protection to hippocampal cells by decreasing neuronal apoptosis during acute ischemia and may be useful in clinical treatment development, pending further assessment of the clinical effects of the drug.

The cell-extrinsic apoptosis pathway Fas $\rightarrow$ FADD $\rightarrow$ caspase- $8 \rightarrow$ caspase- 3 is activated by binding of pro-apoptotic ligands, including Fas/CD95L (TNFSF6) or Apo2L/TRAIL (TNFSF10) to their cognate death domain-containing receptors on the surface of target cells (20-25). In a previous study, 
Table II. Effects of acetylpuerarin on caspase-3 and caspase- 8 activities (24-h reperfusion).

OD, mg

\begin{tabular}{lccc}
\cline { 3 - 4 } Group & Acetylpuerarin concentration, $\mu \mathrm{M}$ & Caspase-3 & Caspase- 8 \\
\hline OGD/R & 0 & $4.36 \pm 0.26$ & $4.27 \pm 0.29$ \\
OGD/R + acetylpuerarin $^{\mathrm{a}}$ & & & \\
VII & 0.1 & $3.78 \pm 0.13^{\mathrm{b}}$ & $3.40 \pm 0.28^{\mathrm{c}}$ \\
VIII & 0.4 & $3.14 \pm 0.26^{\mathrm{b}}$ & $2.80 \pm 0.32^{\mathrm{c}}$ \\
IX & 1.6 & $2.28 \pm 0.27^{\mathrm{b}}$ & $2.55 \pm 0.23^{\mathrm{c}}$ \\
\hline
\end{tabular}

${ }^{a}$ Treated with $3 \mathrm{~h}$ oxygen-glucose deprivation and $24 \mathrm{~h}$ reperfusion. ${ }^{\mathrm{b}} \mathrm{P}<0.05$, vs. caspase- 3 activity in the OGD/R $24 \mathrm{~h}$ group. ${ }^{\mathrm{c}} \mathrm{P}<0.05$, vs. caspase- 8 activity in the OGD/R 6 h group. Values are expressed as the mean \pm SD of three independent experiments. OGD/R, oxygen-glucose deprivation/reperfusion.

A
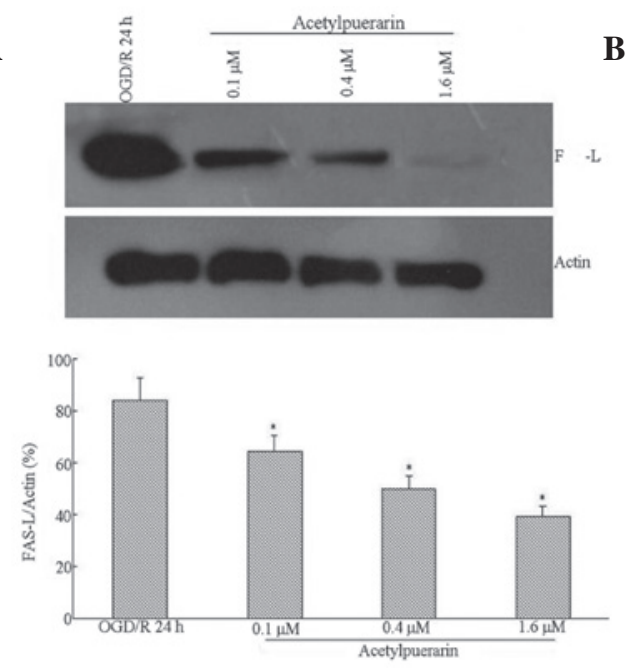

B
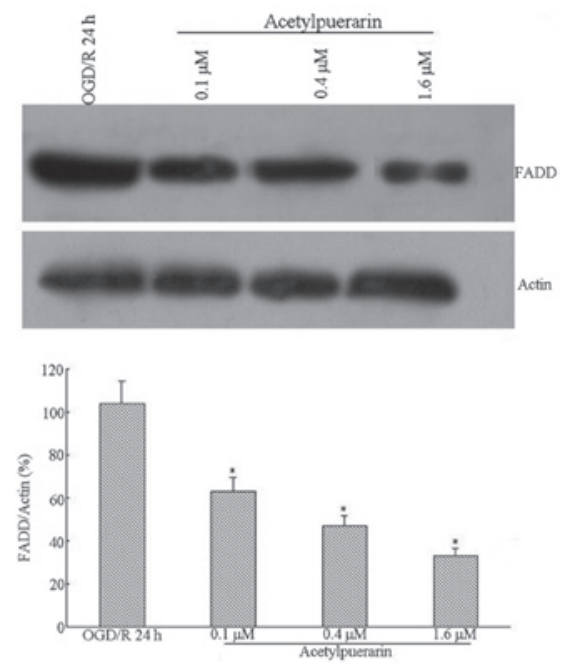

C
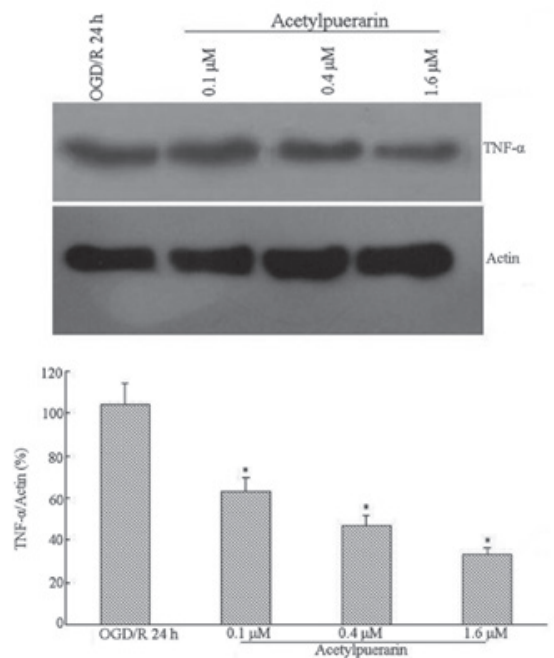

Figure 4. Effects of acetylpuerarin on OGD/R-induced Fas-L, TNF- $\alpha$ and FADD protein expression. Relative levels of (A) Fas-L, (B) FADD and (C) TNF- $\alpha$ in hippocampal neurons were determined by western blot analysis. $\beta$-actin was used as a protein loading control. Values are expressed as the mean \pm SD of three independent experiments. "P<0.05 vs. OGD/R 24 h. OGD/R, oxygen-glucose deprivation/reperfusion; Fas-L, Fas-ligand; TNF- $\alpha$, tumor necrosis factor- $\alpha$; FADD, Fas-associated death domain.

acetylpuerarin was shown to alleviate morphological damage and increase neuron viability, consistent with the current findings (5). The current study is also consistent with reports indicating that TNF- $\alpha$ activation, followed by the inhibition of subsequent inflammatory responses, apoptosis formation (active caspase-3) and neutrophil activation are central to the 
neuroprotective action of puerarin, which may be hypothesized to be similar to those of its derivative, acetylpuerarin (15). Furthermore, the patterns of cell loss following OGD/R are likely to depend on the severity of injury (19), a hypothesis supported by the current observations that changes in DAPI and TUNEL-positive cells (apoptosis) at $24 \mathrm{~h}$ occurred independent of acetylpuerarin treatment concentrations. Thus, acetylpuerarin acts on several of the key mechanisms that affect apoptosis rate during cerebral ischemia.

In the case of clinical ischemic stroke, the total ischemic lesion consists of a penumbral field, which is the most common site of cell death and a core that is the central site to necrosis (20). While rapid damage to the core may not be easily avoided (21), subsequent cell death in the penumbral field over following days and weeks may be mediated by medications, which prevent apoptosis (22). Increasing inflammatory cytokine levels following OGD/R are mechanistically associated with cell death following the ischemic episode. Specifically, upregulation of OGD-induced TNF- $\alpha$ may increase binding to the TNF receptor I (TNFRI) and TNFRII, inducing receptor oligomerization and upregulating recruitment of other cytoplasmic signaling proteins (23). Since acetylpuerarin effectively and consistently reduces hippocampal neuron apoptosis in the current study and in a previous study (5), it may therefore, present an effective neuroprotection strategy, meriting further clinical investigation. In addition, the current study demonstrated that Fas and its only endogenous ligand, Fas-L, factors which are linked to the reduction of apoptosis signaling in ischemic stroke patients, were downregulated, $(24,25)$. Thus, acetylpuerarin may have additional downstream effects on the TNFRI-associated death domain protein (TRADD). Notably, reductions in the signaling molecules Fas and FADD, and reduced subsequent activation of caspase- 8 , further suggested that TRADD was downregulated by acetylpuerarin treatment following OGD $(23,26)$. Thus, acetylpuerarin may confer neuroprotection by mediating OGD-induced TNF- $\alpha$ and thus, downregulating a number of downstream inflammatory signaling molecules, ultimately reducing apoptosis.

Since acetylpuerarin treatment inhibited capsase- 8 activation, it may also reduce mitochondrial-dependent and -independent apoptosis, characterized, respectively, by conversion of the inactive cytosolic form of Bid, a 'BH3-only' pro-apoptosis Bcl-2 family protein, into a truncated fragment (tBid), followed by mitochondrial translocation (27) and activation of caspase-3, the 'executioner caspase' by caspase-8 (28). Truncated tBid also appears to have increased affinity for anti-apoptotic Bcl-2, as well as for Bax/Bak-like proteins, causing Bax/Bak oligomerization and cytochrome $c$ release. Released cytochrome $c$ binds to apoptotic protease-activating factor- 1 and in turn triggers caspase- 9 activation. This apical caspase activates one or more downstream caspases, including caspases-3, -6 and -7 , causing apoptotic cell death (29). It has also been suggested that activation of the apoptosis-triggering enzyme caspase-8 reaches a maximum at $6 \mathrm{~h}$ following the OGD/R insult (19), consistent with the current increased caspase- 8 spectrophotometric detection assay findings. Thus, by inhibiting caspase- 8 and -3 activities, acetylpuerarin prevents neuronal apoptosis through two distinct and well-described mechanisms.
In considering these results, it is important to consider that in vivo results may vary and that systemic side effects may be associated with treatment. Thus, the effects of acetylpuerarin in vivo merit further clinical investigation. Since the effects of acetylpuerarin were examined over a relatively short $24 \mathrm{~h}$ period following reperfusion, it is also important to determine, in future studies, whether acetylpuerarin is capable of mediating neuronal death and promoting functional neurological recovery over longer periods, a current topic of research in our laboratory.

The current findings demonstrated that acetylpuerarin treatment induced dose-dependent neuroprotection against OGD/R-induced neuronal cell death by inhibiting Fas-L, FADD and TNF- $\alpha$ pathway-mediated apoptosis. Furthermore, caspase- 3 and -8 activities were inhibited, providing further evidence that acetylpuerarin impacts a number of pathways known to induce apoptosis following OGD/R. Thus, acetylpuerarin may be a promising future candidate for the clinical treatment of acute ischemic stroke, pending further mechanistic verification and clinical investigations.

\section{Acknowledgements}

This study was supported by a grant from the Nature Science Foundation of Shandong province (no. ZR2010HM132).

\section{References}

1. Truelsen T, Begg S and Mathers C: The global burden of cerebrovascular disease. Global Burden of Disease 2000. World Health Organization, Geneva, 2000.

2. Zhao DX, Feng J, Cong SY and Zhang W: Association of E-selectin gene polymorphisms with ischemic stroke in a Chinese Han population. J Neurosci Res 90: 1782-1787, 2012.

3. Wise-Faberowski L, Raizada MK and Sumners C: Oxygen and glucose deprivation-induced neuronal apoptosis is attenuated by halothane and isoflurane. Anesth Analg 93: 1281-1287, 2001.

4. Lu Y, Zhang J, Ma B, et al: Glycine attenuates cerebral ischemia/reperfusion injury by inhibiting neuronal apoptosis in mice. Neurochem Int 61: 649-658, 2012.

5. Liu R, Wei XB and Zhang XM: Effects of acetylpuerarin on hippocampal neurons and intracellular free calcium subjected to oxygen-glucose deprivation/reperfusion in primary culture. Brain Res 1147: 95-104, 2007.

6. No authors listed: Tissue plasminogen activator for acute ischemic stroke. The National Institute of Neurological Disorders and Stroke rt-PA Stroke Study Group. N Engl J Med 333: 1581-1587, 1995.

7. Elijovich L and Chong JY: Current and future use of intravenous thrombolysis for acute ischemic stroke. Curr Atheroscler Rep 12: 316-321, 2010.

8. Wardlaw JM, Warlow CP and Counsell C: Systematic review of evidence on thrombolytic therapy for acute ischaemic stroke. Lancet 350: 607-614, 1997.

9. Hou L, Wei XB, Li XM, Zhong Y, Zuo CX and Zhang XM: Protective effects of acetylpurarin on focal brain ischemia-reperfusion injury in rats. Chin Pharm J 42: 1469-1472, 2007 (In Chinese).

10. Tan Y, Liu M and Wu B: Puerarin for acute ischemic stroke. Cochrane Database Syst Rev 2008: CD004955, 2008.

11. Wu B, Liu M, Liu H, et al: Meta-analysis of traditional Chinese patent medicine for ischemic stroke. Stroke 38: 1973-1979, 2007.

12. Xu X, Zhang S, Zhang L, Yan W and Zheng X: The neuroprotection of puerarin against cerebral ischemia is associated with the prevention of apoptosis in rats. Planta Med 71: 585-591, 2005.

13. Hou L, Zhang XM and Wei XB: Protective effects of Compounds N-2211 on focal brain ischemia-reperfusion injury in rats. Qilu Pharm Affairs 23: 52-54, 2004 (In Chinese).

14. Li XM, Wei XB, Zhang XM, Hou L, Zhong Y and Zuo CX: Effect of acetylpuerarin on NO level and NOS activity in brain tissue and serum of focal cerebral ischemia reperfusion injury rats. Chin Pharm J 11: 829-832, 2005. 
15. Lou HY, Wei XB, Zhang B, Sun $X$ and Zhang XM: Hydroxyethylpuerarin attenuates focal cerebral ischemia-reperfusion injury in rats by decreasing TNF-alpha expression and NF-kappaB activity. Yao Xue Xue Bao 42: 710-715, 2007.

16. Yao YY, Liu DM, Xu DF and Li WP: Memory and learning impairment induced by dexamethasone in senescent but not young mice. Eur J Pharmacol 574: 20-28, 2007.

17. Ziu M, Fletcher L, Rana S, Jimenez DF and Digicaylioglu M: Temporal differences in microRNA expression patterns in astrocytes and neurons after ischemic injury. PLoS One 6: e14724, 2011.

18. Sreelatha A, Jeyachitra A and Padma PR: Antiproliferation and induction of apoptosis by Moringa oleifera leaf extract on human cancer cells. Food Chem Toxicol 49: 1270-1275, 2011.

19. Badiola N, Malagelada C, Llecha N, et al: Activation of caspase- 8 by tumour necrosis factor receptor 1 is necessary for caspase-3 activation and apoptosis in oxygen-glucose deprived cultured cortical cells. Neurobiol Dis 35: 438-447, 2009.

20. Yao H, Takasawa R, Fukuda K, et al: DNA fragmentation in ischemic core and penumbra in focal cerebral ischemia in rats. Brain Res Mol Brain Res 91: 112-118, 2001.

21. Barone FC and Feuerstein GZ: Inflammatory mediators and stroke: new opportunities for novel therapeutics. J Cereb Blood Flow Metab 19: 819-834, 1999.
22. Jiang $Y, W u$ J, Keep RF, Hua Y, Hoff JT and Xi G: Hypoxia-inducible factor-1alpha accumulation in the brain after experimental intracerebral hemorrhage. J Cereb Blood Flow Metab 22: 689-696, 2002.

23. Chen $\mathrm{G}$ and Goeddel DV: TNF-R1 signaling: a beautiful pathway. Science 296: 1634-1635, 2002.

24. Liu L, Kim JY, Koike MA, et al: FasL shedding is reduced by hypothermia in experimental stroke. J Neurochem 106: 541-550, 2008.

25. Ruan W, Lee CT and Desbarats J: A novel juxtamembrane domain in tumor necrosis factor receptor superfamily molecules activates Rac1 and controls neurite growth. Mol Biol Cell 19: 3192-3202, 2008.

26. Ashkenazi A and Dixit VM: Death receptors: signaling and modulation. Science 281: 1305-1308, 1998.

27. Luo X, Budihardjo I, Zou H, Slaughter C and Wang X: Bid, a $\mathrm{Bcl} 2$ interacting protein, mediates cytochrome $c$ release from mitochondria in response to activation of cell surface death receptors. Cell 94: 481-490, 1998.

28. Varfolomeev EE and Ashkenazi A: Tumor necrosis factor: an apoptosis JuNKie? Cell 116: 491-497, 2004

29. Garrido C, Gurbuxani S, Ravagnan L and Kroemer G: Heat shock proteins: endogenous modulators of apoptotic cell death. Biochem Biophys Res Commun 286: 433-442, 2001. 